\title{
O Poder dos Caminhos Quase Mais Curtos e o Impacto da Mobilidade dos Nós em Redes Dinâmicas
}

\author{
Dianne S. V. Medeiros ${ }^{1}$, Miguel Elias M. Campista ${ }^{1}$ \\ ${ }^{1}$ Universidade Federal do Rio de Janeiro - PEE-COPPE/Del-Poli/GTA \\ \{dianne, miguel\}@gta.ufrj.br
}

\begin{abstract}
Resumo. Este trabalho investiga o impacto da mobilidade dos nós na transferência de dados em múltiplos saltos, o efeito do uso de caminhos um pouco maiores do que os mais curtos na importância relativa dos nós e o desempenho da rede na presença de falha em nós centrais. Para analisar o primeiro aspecto, propõe-se a $(\kappa, \lambda)$-vizinhança, que estende o conceito de vizinhança para incluir nós a múltiplos saltos, restringindo o estabelecimento de enlaces de acordo com a velocidade relativa entre os nós. Os resultados obtidos são usados no desenvolvimento de três estratégias de encaminhamento, que resultam em uma redução significativa do consumo de recursos, sem provocar grande impacto na taxa média de entrega de pacotes. O segundo aspecto é analisado através da intermediação $\rho$-geodésica, uma métrica de centralidade proposta neste trabalho, que usa outros caminhos além dos mais curtos para quantificar a importância relativa dos nós. O custo desses caminhos é limitado por um fator de espalhamento $\rho$. O terceiro aspecto é analisado através de simulações nas quais são provocadas falhas em nós centrais. Esses nós, quando classificados segundo a métrica proposta, estão em posições menos críticas em relação à conectividade da rede. Logo, os nós mais centrais de acordo com a intermediação $\rho$-geodésica são mais resilientes a falhas de outros nós. Isso é essencial para determinar o papel que cada nó deve exercer para a rede.
\end{abstract}

\section{Motivação, Objetivos e Trabalhos Relacionados}

A mobilidade dos nós em redes sem fio é estudada há muito tempo. No entanto, continua sendo um tópico de pesquisa em aberto, principalmente devido ao surgimento de novos paradigmas, como as Redes Ad Hoc Veiculares (VANETs - Vehicular Ad Hoc Networks). Nessas redes, os modelos de mobilidade e de comunicação desenvolvidos para as Redes Ad Hoc Móveis (MANETs - Mobile Ad Hoc Networks) não são adequados, devido à diferença na dinâmica dos nós que as compõem. Além disso, a complexidade da comunicação nas VANETs aumenta ainda mais à medida que mais saltos são necessários para alcançar um destino. Em redes sem fio estáticas, os protocolos desenvolvidos consideram que a falta de caminhos fim-a-fim é transitória, mas isso não é necessariamente verdade nas VANETs. A probabilidade de a rede não ser conexa é mais alta, de forma que as oportunidades de contato devem ser eficientemente aproveitadas. Para tanto, quanto mais o protocolo utilizado for capaz de prever quando as oportunidades acontecem, quanto tempo duram e qual a largura de banda disponível, melhor. Assim, é necessário realizar novos estudos e propor modelos mais apropriados às características das novas redes.

Para vencer os desafios das VANETs, diversos trabalhos estudam os padrões de mobilidade e de conectividade entre os nós [Gaikwad and Zaveri 2011, Spaho et al. 2011, 
Madi and Al-Qamzi 2013], enquanto outros propõem mecanismos complexos para prever a disponibilidade [Rezende et al. 2009] e a interrupção [Taleb et al. 2007] de contatos a um salto de distância. Isso permite que os nós obtenham conhecimento sobre o comportamento da sua própria vizinhança [Conan et al. 2007, Passarella and Conti 2011, Phe-Neau et al. 2012, Phe-Neau et al. 2013, Hoque et al. 2014] e do histórico de mobilidade [Shelly and Babu 2017] e, assim, possam decidir a cada encontro qual a melhor ação a ser tomada para aumentar a probabilidade de entrega de mensagens. Dentre os autores que se dedicam ao estudo da vizinhança, Phe-Neau et al. [Phe-Neau et al. 2012, Phe-Neau et al. 2013] propõem o uso de uma vizinhança estendida, que incorpora nós à vizinhança de um nó mesmo quando estão a múltiplos saltos de distância. Apesar de utilizarem informações de mobilidade, nenhum desses trabalhos estuda explicitamente a relação entre a velocidade relativa dos nós e o número de saltos que os separam, nem a utilidade dos contatos estabelecidos.

A qualidade da comunicação em cenários dinâmicos depende fortemente da habilidade dos nós para estabelecer rotas eficientes de acordo com a condição atual da rede [Campista et al. 2008]. Essas condições também ditam a importância de um nó para a rede como um todo e podem ser medidas através de métricas de centralidade, como o grau, a proximidade e a intermediação [Freeman 1978, Hui et al. 2008, Wehmuth and Ziviani 2013]. Essa última se relaciona com o controle que um nó exerce sobre o fluxo entre outros nós [Shaw 1954], e é foco deste trabalho. A quantificação da importância de um nó é relevante para a análise de redes porque permite uma melhor compreensão da dinâmica das relações entre os nós, que pode levar a uma definição mais adequada das funções e papeis exercidos por eles. Em redes de computadores, por exemplo, pode ser conveniente que analisadores de fluxo sejam instalados em nós com elevada centralidade de intermediação, uma vez que é mais provável que eles participem da comunicação entre outros nós.

A intermediação tradicional utiliza apenas os caminhos mais curtos para definir a importância de um nó. Porém, fluxos nem sempre seguem esses caminhos [Newman 2005], como rumores e doenças, que se espalham aleatoriamente [Stephenson and Zelen 1989]. Assim, existem na literatura, diversas variantes da centralidade de intermediação tradicional, como a intermediação por percursos aleatórios [Newman 2005], que considera que os fluxos se movem aleatoriamente, passando por todos os caminhos existentes, e a escalonada por distância [Borgatti and Everett 2006], que considera apenas os caminhos mais curtos, ponderados pelo comprimento total do caminho. O uso de múltiplos caminhos, mesmo que não sejam tão curtos quanto os mais curtos, pode fornecer resiliência à rede e aumentar a vazão total. Ao considerar outros caminhos além dos mais curtos, nenhum dos trabalhos encontrados limita o número nem o custo dos caminhos.

Este trabalho se preocupa em investigar dois temas importantes para a análise de redes com topologias dinâmicas.

Problema 1: Influência da velocidade relativa nos contatos em múltiplos saltos: investiga o impacto da mobilidade dos nós no estabelecimento de comunicações em múltiplos saltos através da análise da vizinhança estendida. 
Problema 2: Avaliação da importância dos nós: atribui importância a nós que não participam ou pouco participam de caminhos mais curtos, considerando que os fluxos não seguem caminhos aleatórios na rede e que caminhos muito longos não devem ter grande influência por concentrarem menos fluxos [Newman 2005].

\section{Contribuições da Tese}

As contribuições principais deste trabalho trazem inovações que foram reconhecidas pela comunidade científica, resultando em publicações em revistas internacionais [Medeiros et al. 2018, Medeiros et al. 2017b], e congressos nacionais [Hernandez et al. 2015, Medeiros et al. 2016a, Medeiros et al. 2017a] e internacionais [Medeiros et al. 2016b].

As contribuições reportadas agregam mais conhecimento para melhorar a compreensão da dinâmica da rede e dos papeis desempenhados pelos nós, além de fornecer informações que permitem desenvolver protocolos de encaminhamento de mensagens mais eficientes. Essas contribuições estão sumarizadas a seguir. (i) Identifica-se a importância da análise da influência da velocidade relativa nas comunicações em redes de múltiplos saltos, e a necessidade de uma métrica de intermediação que capture de forma adequada a importância de nós que participam de caminhos um pouco maiores do que o mais curto. (ii) Propõe-se a definição estendida de vizinhança do nó para incluir os nós a múltiplos saltos de distância do nó focal, restringindo o estabelecimento de enlaces de acordo com suas velocidades relativas. (iii) A vizinhança estendida é caracterizada em três cenários distintos, avaliando-se a utilidade de cada oportunidade de contato para transferência de arquivos grandes. Demonstra-se que o consumo de recursos na rede pode ser reduzido sem diminuir a taxa média de entrega de pacotes ao se utilizar os resultados encontrados na análise de vizinhança para a tomada de decisões de encaminhamento. (iv) Propõe-se também a intermediação $\rho$-geodésica, que atribui importância a nós que participam de outros caminhos além dos mais curtos. (v) Essa métrica é caracterizada através de investigações comparativas com outras três métricas de intermediação. O efeito mais significativo é a reclassificação dos nós, que identifica centralidade em posições menos críticas em termos de conectividade da rede. Isso significa que os nós considerados mais importantes são resilientes a falhas de outros nós e, ainda assim, possuem papel central. Os desdobramentos são diversos, incluindo o emprego desses nós para desempenharem funções importantes de rede.

\section{Proposta}

Este trabalho propõe a $(\kappa, \lambda)$-vizinhança, que estende a vizinhança de um nó para considerar as velocidades relativas; e a centralidade de intermediação $\rho$-geodésica, capaz de atribuir importância a nós que não participam de outros caminhos além dos mais curtos.

\subsection{Problema 1 e a $(\kappa, \lambda)$-vizinhança}

A vizinhança estendida proposta por Phe-Neau et al. [Phe-Neau et al. 2012, Phe-Neau et al. 2013] inclui nós que estão a múltiplos saltos de distância do nó central da vizinhança. Este trabalho agrega mais uma variável a essa vizinhança, utilizando a velocidade relativa dos nós para reger o estabelecimento dos enlaces entre nós adjacentes. O grafo da rede pode ser dividido em subgrafos de acordo com a faixa de 


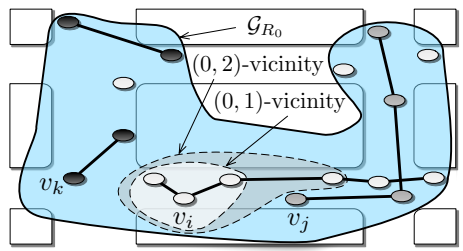

(a) $\mathcal{G}_{R_{0}}, \quad(0,1)-\quad$ e $(0,2)-$ vizinhanças, baixa velocidade relativa.

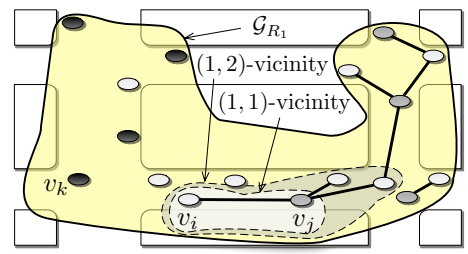

(b) $\mathcal{G}_{R_{1}}, \quad(1,1)-\quad$ e $(1,2)-$ vizinhanças, média velocidade relativa.

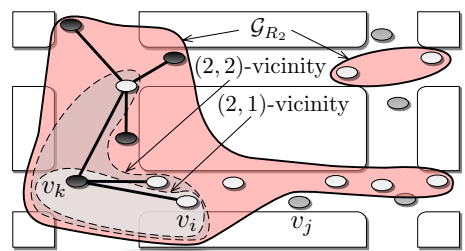

(c) $\mathcal{G}_{R_{2}}, \quad(2,1)-$ e $(2,2)-$ vizinhanças, alta velocidade relativa.

Figura 1. Exemplo de $(\kappa, \lambda)$-vizinhanças de um nó $v_{i}$ nos subgrafos $\mathcal{G}_{R_{\kappa}}$ gerados ao se considerar enlaces existentes apenas entre nós com velocidades relativas em uma subfaixa de velocidades, $R_{\kappa}$.

velocidades relativas considerada. Esse conceito está representado na Figura 1, onde a cor dos nós indica a velocidade absoluta escalar, quanto mais escuro mais rápido o nó se move. Se a velocidade relativa não for considerada, existe apenas um grafo $\left(\mathcal{G}_{R_{\kappa}}=\mathcal{G}_{R_{0}}\right)$, que inclui todos os nós da rede. Desde que estejam sob alcance rádio mútuo, um enlace entre dois nós adjacentes existe. Sequências de $\lambda+1$ nós adjacentes a partir de um nó focal interligados por seus enlaces representam a vizinhança de $\lambda$ saltos daquele nó. Dividindo a faixa de velocidades relativas em 3 subfaixas, o grafo passa a ser composto de 3 subgrafos (velocidades relativas baixas, médias e altas), nos quais um enlace existe apenas se a velocidade relativa entre os nós estiver dentro da subfaixa $\kappa$, resultando em alterações na vizinhança do nó focal. A análise do impacto dessa vizinhança no estabelecimento de contatos é feita utilizando três conjuntos de dados distintos.

Os resultados da investigação do impacto da $(\kappa, \lambda)$-vizinhança nos contatos entre os nós são usados para avaliar três propostas de estratégias de encaminhamento que impõem restrições tomadas apenas pelo nó de origem. As estratégias foram implementadas no Network Simulator 3 (NS-3), tendo como base a operação do OLSR e estão resumidas a seguir.

- Encaminhamento Restrito por Velocidades Relativas (RelSpeedR): o nó de origem restringe o encaminhamento para o próximo salto de acordo com a velocidade relativa entre eles. Com essa velocidade relativa, se for provável que a mensagem chegue ao destino, ela é encaminhada, caso contrário é descartada.

- Encaminhamento Restrito por Vizinhança (VicR): durante a construção da vizinhança de um salto, o nó verifica sua velocidade relativa com cada um dos nós vizinhos e suprime os enlaces para os quais a velocidade relativa não está na faixa que permite comunicações a um salto de distância. O restante do encaminhamento ocorre normalmente como no OLSR.

- Encaminhamento Restrito por Velocidade Relativa e Vizinhança (RelSpeedVicR): utiliza os dois modos anteriores simultaneamente.

\subsection{Problema 2 e a intermediação $\rho$-geodésica}

Este trabalho propõe a centralidade de intermediação $\rho$-geodésica para avaliar a importância de nós que não participam ou pouco participam dos caminhos mais curtos, utilizando os conceitos de espalhamento e caminhos quase mais curtos. $\mathrm{O}$ fator de espalhamento $\rho$ limita a busca por caminhos no grafo da rede, evitando a explosão do número 
de caminhos. Os caminhos quase mais curtos são aqueles que não ultrapassam um limite determinado pelo espalhamento da seguinte forma: $\delta_{i, j} \leq \delta_{i, j}^{*}+\rho$, onde $\delta_{i, j}$ é o custo do caminho quase mais curto entre os nós $v_{i}$ e $v_{j}, \delta_{i, j}^{*}$ é o custo do caminho mais curto e $\rho$ é o espalhamento. A métrica proposta computa a frequência de participação de um nó nos caminhos mais curtos e quase mais curtos, e pondera esse valor de acordo com o custo adicional por utilização de caminhos quase mais curtos. Dessa forma, reduz-se o peso da contribuição do caminho quando ele é muito mais custoso do que o caminho mais curto, uma vez que a informação tende a se concentrar em caminhos mais curtos [Stephenson and Zelen 1989]. A métrica proposta é formalizada na Equação 1, onde $n_{i, j}$ e $n_{i, j}^{*}$ são os números de caminhos quase mais curtos e mais curtos entre $v, v_{j}$, respectivamente; $n_{i, j}\left(v_{k}\right)$ e $n_{i, j}^{*}\left(v_{k}\right)$ são os que passam por $v_{k}$. Nessa proposta, um nó é considerado mais central quando participa de mais caminhos, sejam eles mais curtos ou quase mais curtos, priorizando a participação em caminhos mais curtos. Note que $\rho=0$ recai na intermediação tradicional.

$$
B_{\rho}\left(v_{k}\right)=\sum_{\substack{i \in \mathcal{V} \\ \delta_{i, k}+\delta_{k, j}-\delta_{i, j}^{*} \leq \rho}} \frac{n_{i, j}^{*}\left(v_{k}\right)+n_{i, j}\left(v_{k}\right)}{n_{i, j}^{*}+n_{i, j}} \times \frac{\delta_{i, j}^{*}}{\delta_{i, k}+\delta_{k, j}} .
$$

A métrica proposta é caracterizada através de comparações com as centralidades de intermediação tradicional, por percursos aleatórios e escalonada por distância. A ideia da intermediação $\rho$-geodésica difere da intermediação por percursos aleatórios por considerar que os fluxos não viajam aleatoriamente pela rede até encontrar o destino, e limita o custo dos caminhos utilizados, uma vez que os fluxos tendem a se concentrar nos caminhos mais curtos. Isso leva a uma redução na complexidade da métrica: $\mathrm{O}\left(n^{3}\right)$, contra $\mathrm{O}\left(n^{4}\right)$ da por percursos aleatórios, no pior caso.

\section{Resultados}

Essa seção discute os resultados mais relevantes e está dividida de acordo com o problema.

\subsection{Problema 1: Influência da velocidade relativa nos contatos em múltiplos saltos}

As análises realizadas confirmam que a distância em saltos, o número e a duração dos contatos são afetadas negativamente pelo aumento da velocidade relativa. Dessa forma, quanto maior for essa velocidade, menor é a probabilidade de serem estabelecidos contatos duradouros e em múltiplos saltos. Mais importante, do que o número e a duração dos contatos, é a utilidade de um contato. Essa utilidade pode ser determinada de acordo com a aplicação em uso. Aplicações de entretenimento, por exemplo, podem exigir que grandes volumes de dados sejam transferidos em um único contato. Neste trabalho, um contato é considerado útil se permitir a transferência de pelo menos $1 \mathrm{MB}$ de dados, usando a menor taxa de transmissão da tecnologia (3 Mb/s para o IEEE 802.11p). Os resultados mostram que o número de contatos capazes de transferir esses dados é mais numeroso para distâncias em salto curtas com velocidades relativas baixas, e torna-se menor para velocidades relativas altas e número de saltos maior. Apesar disso, 4\% dos contatos conseguem transferir dados em até 8 saltos de distância em altas velocidades no cenário mais denso. Observa-se também que, em geral, a velocidade relativa reduz mais severamente o número de contatos úteis do que o aumento da distância em saltos. Os resultados das 
análises indicam que, apesar da influência da mobilidade dos nós na conectividade da rede, também é possível explorar contatos em distâncias mais longas e múltiplos saltos, desde que ocorram em baixas velocidades relativas.

Dados os resultados obtidos, três estratégias de encaminhamento foram propostas, VicR, RelspeedVicR e Relspeed (Seção 3). Elas modificam o OLSR, incluindo a velocidade absoluta de cada nó nos pacotes Hello, o algoritmo de descoberta de vizinhança e de encaminhamento dos dados. A análise realizada mostra que as estratégias propostas são capazes de reduzir a taxa de encaminhamento de pacotes descartados mantendo-se a mesma taxa de entrega de pacotes. Isso representa uma redução no desperdício de recursos, devido à diminuição da ocupação da rede com dados que não chegam ao destino, mesmo utilizando um protocolo que não foi desenvolvido para as especificidades das redes veiculares, incrementado apenas com os resultados deste trabalho.

\subsection{Problema 2: Avaliação da importância dos nós}

O impacto e a relevância da intermediação $\rho$-geodésica são analisados aplicando a métrica a quatro conjuntos de dados distintos, usando $\rho \leq 5$, com $\rho \in \mathbb{N}$. Os resultados mostram que a intermediação $\rho$-geodésica, a por percursos aleatórios e a escalonada por distância são capazes de modificar o ranqueamento produzido pela intermediação tradicional. Apesar disso, o coeficiente W de Kendall (Figura 2) mostra que o nível de concordância entre as métricas é elevado. Isso significa que as métricas estão medindo os mesmos tipos de características na rede. Porém, essa análise não reflete a taxa com a qual os nós são reclassificados, comparadas à intermediação tradicional, sendo igual à $66,1 \%$ para a intermediação escalonada por distância, $75,8 \%$ para a por percursos aleatórios, e varia entre $74,2 \%$ e $79,0 \%$ para a $\rho$-geodésica $(\rho \leq 5)$.

Nós com elevada intermediação tradicional podem ser bons candidatos para a instalação de analisadores de fluxo, caso eles sigam caminhos mais curtos. Em uma rede dinâmica, esses nós devem liderar o ranqueamento de centralidades pelo maior tempo possível, para que não seja necessário transferir a função para outro nó. Além disso, eles não devem ser pontos de articulação, uma vez que a falha em algum deles levaria ao particionamento da rede. O número de realocações que poderiam ser evitadas ao se classificar os nós segundo a métrica proposta é sempre maior do que utilizando as outras métricas, inclusive para os nós inicialmente mais centrais. Além disso, a intermediação $\rho$-geodésica elege menos pontos de articulação nas posições mais centrais, comparada à intermediação tradicional. Uma falha ocorrida nos nós mais centrais de cada métrica prejudica severamente a vazão da rede, mas a probabilidade de atingir um ponto de articulação é menor ao se classificar os nós de acordo com a métrica proposta, reduzindo,consequentemente, a probabilidade de particionamento da rede.

\section{Considerações Finais}

Este trabalho estudou a influência da estrutura da rede e da mobilidade dos nós no encaminhamento de dados e na avaliação da importância relativa dos nós. Para tal, foram propostos o conceito de $(\kappa, \lambda)$-vizinhança, que inclui a velocidade relativa e o número de saltos na definição da vizinhança de um nó; e uma nova métrica de centralidade, a intermediação $\rho$-geodésica que leva em consideração caminhos mais curtos e quase mais curtos, e o custo desses caminhos em relação aos mais curtos para determinar a importância de um 

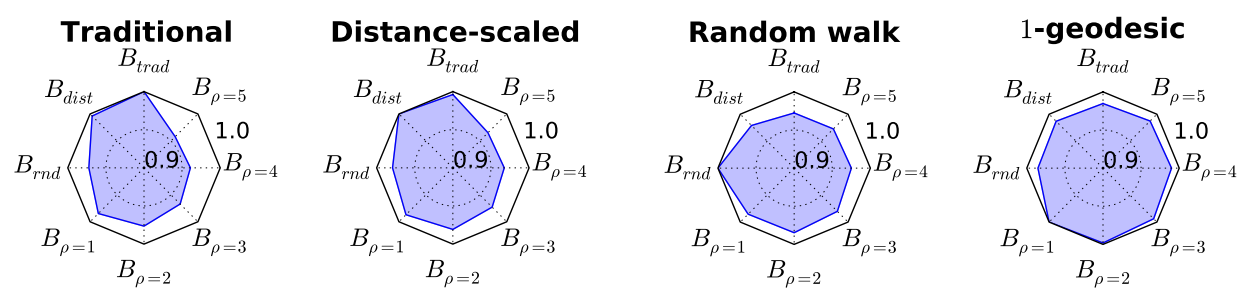

Figura 2. Coeficiente $\mathrm{W}$ de Kendall para cada combinação de métricas mostra que o nível de concordância entre elas é elevado. A menor concordância ocorre entre a intermediação tradicional e a 5-geodésica, devido ao número de caminhos quase mais curtos incluídos na computação da métrica proposta.

nó. Os caminhos quase mais curtos são limitados pelo espalhamento $\rho$, que define o custo adicional em relação aos caminhos mais curtos. Os resultados da análise de vizinhança confirmam a intuição de que contatos tendem a acontecer a poucos saltos de distância e com velocidades relativas baixas. A inclusão dos resultados encontrados no desenvolvimento de três estratégias e encaminhamento salto-a-salto mostrou que é possível reduzir o desperdício de recursos da rede, mantendo-se a mesma taxa de entrega de pacotes. A métrica de centralidade proposta se mostra capaz de reclassificar os nós que participam de poucos ou nenhum caminho mais curto. O efeito mais importante dessa reclassificação é a identificação de nós ainda centrais, mas que ocupam posições menos críticas em relação à conectividade da rede. Os desdobramentos dos estudos realizados são diversos, incluindo o desenvolvimento de protocolos de encaminhamento de mensagens mais eficientes e a agregação de mais conhecimento para uma compreensão mais profunda da dinâmica da rede e dos papeis desempenhados pelos nós. Isso é essencial para selecionar adequadamente as funções exercidas por cada nó para a rede.

\section{Referências}

Borgatti, S. P. e Everett, M. G. (2006). A graph-theoretic perspective on centrality. Social Networks, 28(4):466-484.

Campista, M. E. M., Esposito, P. M., Moraes, I. M., k. Costa, L. H., Duarte, O. C. M. B., Passos, D. G., Albuquerque, C. V. N., Saade, D. C. M., e Rubinstein, M. G. (2008). Routing metrics and protocols for wireless mesh networks. Network, 22(1):6-12.

Conan, V., Leguay, J., e Friedman, T. (2007). Characterizing pairwise inter-contact patterns in delay tolerant networks. In Autonomics, págs. 19:1-19:9.

Freeman, L. C. (1978). Centrality in social networks: Conceptual clarification. Social Networks, 1(3):215-239.

Gaikwad, D. S. e Zaveri, M. (2011). Vanet routing protocols and mobility models: A survey. In Trends in Network and Communications, págs. 334-342.

Hernandez, D. A. B., Medeiros, D. S. V., Campista, M. E. M., e de Castro P. Pedroza, A. (2015). Uma avaliação da influência da velocidade dos nós no estabelecimento de caminhos em redes ad hoc veiculares. In SBRC, págs. 431-444.

Hoque, M. A., Hong, X., e Dixon, B. (2014). Efficient multi-hop connectivity analysis in urban vehicular networks. Vehicular Communications, 1(2):78-90. 
Hui, P., Crowcroft, J., e Yoneki, E. (2008). Bubble rap: Social-based forwarding in delay tolerant networks. In MobiHoc, págs. 241-250.

Madi, S. e Al-Qamzi, H. (2013). A survey on realistic mobility models for vehicular ad hoc networks (VANETs). In ICNSC, págs. 333-339.

Medeiros, D. S. V., Campista, M. E. M., de Amorim, M. D., Mitton, N., e Pujolle, G. (2017a). Eficiência dos caminhos quase mais curtos em redes dinâmicas. In $S B R C$, págs. 544-557.

Medeiros, D. S. V., Campista, M. E. M., Mitton, N., de Amorim, M. D., e Pujolle, G. (2016a). Intermediação por espalhamento: Caminhos quase mais curtos também importam. In SBRC, págs. 967-980.

Medeiros, D. S. V., Campista, M. E. M., Mitton, N., de Amorim, M. D., e Pujolle, G. (2016b). Weighted betweenness for multipath networks. In GIIS, págs. 1-6.

Medeiros, D. S. V., Campista, M. E. M., Mitton, N., de Amorim, M. D., e Pujolle, G. (2017b). The power of quasi-shortest paths: $\rho$-geodesic betweenness centrality. Trans. on Net. Science and Eng., 4(3):187-200.

Medeiros, D. S. V., Hernandez, D. A. B., Campista, M. E. M., e de Castro P. Pedroza, A. (2018). Impact of relative speed on node vicinity dynamics in vanets. Wireless Networks, PP:1-18.

Newman, M. J. (2005). A measure of betweenness centrality based on random walks. Social Networks, 27(1):39-54.

Passarella, A. e Conti, M. (2011). Characterising aggregate inter-contact times in heterogeneous opportunistic networks. In NETWORKING, págs. 301-313.

Phe-Neau, T., Dias de Amorim, M., Campista, M. E. M., e Conan, V. (2013). Examining vicinity dynamics in opportunistic networks. In $P M 2 H W 2 N$, págs. 153-160.

Phe-Neau, T., Dias de Amorim, M., e Conan, V. (2012). Vicinity-based DTN characterization. In MobiOpp, págs. 37-44.

Rezende, C. G., Pazzi, R. W., e Boukerche, A. (2009). An efficient neighborhood prediction protocol to estimate link availability in VANETs. In MobiWAC, págs. 83-90.

Shaw, M. E. (1954). Group structure and the behavior of individuals in small groups. The J. of Psychology, 38(1):139-149.

Shelly, S. e Babu, A. V. (2017). Link residual lifetime-based next hop selection scheme for vehicular ad hoc networks. J. on Wireless Comm. and Net., 2017(1):23:1-23:13.

Spaho, E., Barolli, L., Mino, G., Xhafa, F., e Kolici, V. (2011). Vanet simulators: A survey on mobility and routing protocols. In $B W C C A$, págs. 1-10.

Stephenson, K. e Zelen, M. (1989). Rethinking centrality: Methods and examples. Social Networks, 11(1):1-37.

Taleb, T., Sakhaee, E., Jamalipour, A., Hashimoto, K., Kato, N., e Nemoto, Y. (2007). A stable routing protocol to support ITS services in VANET networks. Trans. on Vehicular Technology, 56(6):3337-3347.

Wehmuth, K. e Ziviani, A. (2013). DACCER: Distributed assessment of the closeness centrality ranking in complex networks. Computer Networks, 57(13):2536-2548. 